\title{
Ultrafast stimulated Raman study of the complete reactive potential energy surface of a photomolecular rotor
}

\author{
Christopher R. Hall ${ }^{1}$, Wesley R. Browne ${ }^{2}$, Ben L. Feringa ${ }^{2} *$ and Stephen R. Meech ${ }^{{ }^{*}}$ \\ ${ }^{1}$ School of Chemistry, University of East Anglia, Norwich Research Park, Norwich NR4 7TJ, UK \\ ${ }^{2}$ Stratingh Institute for Chemistry, University of Groningen, Nijenborgh 4, 9747 AG Groningen, The \\ Netherlands
}

\begin{abstract}
Femtosecond Stimulated Raman Spectroscopy (FSRS) reports on structure and dynamics of excited states. Here we record FSRS for a photomolecular motor in its stable and metastable states, and thus map the entire excited state PES.
\end{abstract}

\section{Introduction}

Light driven molecular motors based on the chiral overcrowded alkene motif convert the energy of incident photons into intramolecular rotational motion.[1] As such they have the potential to act as a power source for the molecular machines of the future. The essential operational features of photomolecular motors are illustrated in Figure 1. The lowest energy<smiles></smiles>

1a (Stable)

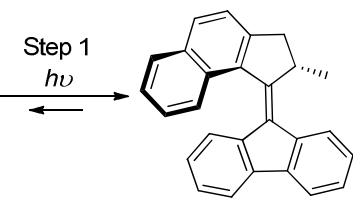

1b (Metastable)
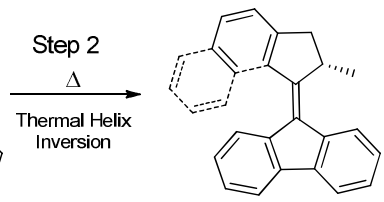

1a (Stable $\left.+180^{\circ}\right)$

Figure 1. Schematic representation of light abd thermally driven transitions between 1a and $\mathbf{1 b}$ states.

ground state conformation (1a) comprises a 'stator' linked via a double bonded 'axle' connected to a 'rotor', which has a chiral centre. The molecule is nonplanar due to steric strain. Excitation of the lowest energy $\pi$ to $\pi *$ transition localised on the axle reduces the bond order, releasing steric strain and hence a barrierless structural relaxation. The result of this excited state structure change is an isomerization reaction leading ultimately to population of the metastable ground state $\mathbf{1 b}$ with a yield of $5-20 \%$ depending on the substituents.[2] For these motors, and those which are symmetrically substituted on the fluorene ring, the conformation $\mathbf{1 b}$ can relax to $\mathbf{1 a}$ by a ground state thermal isomerization either in the reverse or the forward direction, but the forward helix inversion step is overwhelmingly favoured due to a lower energy barrier, leading to a half rotation and 
population of $1 \mathrm{a}$ but rotated by $180^{\circ}$ (Figure 1). Absorption of a second photon followed by a second forward helix inversion completes one cycle of rotary motion. Careful optimisation of the molecular structure has led to motors of the general structure 1a with rotation rates in the $\mathrm{MHz}$ frequency range. Although progress has been rapid, many challenges remain before such motors can form components of functioning molecular machines, including: more detailed understanding of the structure change accompanying excited state reaction; understanding of how that structure change couples to its molecular environment; knowledge and control of the quantum yield of the photochemical step. Each of these requires an accurate picture of the excited state potential energy surface and its coupling to the ground state.

Previously we characterised the excited state dynamics of 1a by ultrafast Time-Resolved Fluorescence (TRF), Transient Absorption (TA) and Femtosecond Stimulated Raman Spectroscopy (FSRS).[2,3] The initial Franck-Condon excited state was observed to relax in ca $100 \mathrm{fs}$ to a dark state which undergoes fast intramolecular vibrational cooling and subsequent picosecond excited state relaxation, leading to formation of a vibrationally hot 1b ground state. The excited state dynamics of this $\mathbf{1 b}$ state are also critical, as its fate determines the positon of the photostationary state, which in-turn connects to the efficiency of the motors. Thus far this state has not been probed experimentally, although it has been the subject of a number of high quality quantum chemical calculations. [4,5].

In this work we extend our ultrafast TA and FSRS experiments to investigate excited state dynamics of $\mathbf{1 b}$ yielding the essential experimental data against which to test theoretical calculations. We find that the excited state lifetime for the dark state which is intermediate between $\mathbf{1 a}$ and $\mathbf{1 b}$, is a function of the initial Franck-Condon excited state. This suggests that the forward and reverse reaction coordinates are distinct. The FSRS data point to significantly different vibrational spectra for the two dark states accessed by the different routes. These data are discussed in the context of the recent quantum chemical calculations, and point to the need for further calculation.

\section{Measurements}

TA and FSRS measurements are made using the same source, a $1 \mathrm{kHz}$ amplified titanium sapphire laser pumping two OPAs, which each output $100 \mathrm{fs}$ tuneable pulses, and a narrowband OPA pumped by picosecond duration $400 \mathrm{~nm}$ pulses generated by second harmonic bandwidth compression. The latter provides the tuneable Raman pump for FSRS measurements. One of the $100 \mathrm{fs}$ OPA is used for the actinic pulse (to generate the $\mathbf{1 b}$ excited state), while the other (or alternatively a fraction of the $800 \mathrm{~nm}$ amplifier output) generates the white light continuum in a sapphire plate. The final temporal resolution is about $100 \mathrm{fs}$, with spectral resolution at $15 \mathrm{~cm}^{-1}$. The $\mathbf{1 b}$ state was generated from $\mathbf{1 a}$ by steady state illumination at $380 \mathrm{~nm}$. By tuning the excitation OPA to $450 \mathrm{~nm}$ we selectively excite 1b from the photostationary state. The Raman pump wavelength was tuned to be resonant with the dark state transient absorption. FSRS were measured on the low wavenumber 'Stokes' side, and observed to be independent of Raman pump wavelength within the band; a Raman pump on the blue side of the transient absorption gave noticeably more complex lineshapes, though the same frequencies

\section{Results and Discussion}

The principle results are shown in Figure 2, where a $\mathrm{CN}$ derivative of the motor has been studied as it has a longer dark state lifetime.[2] Essentially the same results are obtained for the unsubstituted motor, as described in detail elsewhere, but the $\mathbf{1 b}$ decay was too fast for FSRS measurement.[6] 

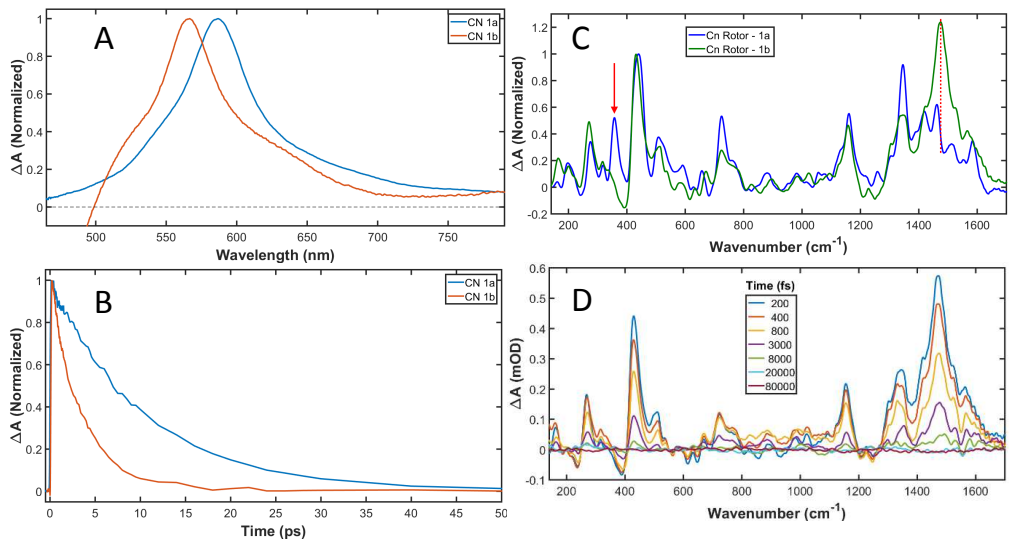

Figure 2. A. TA data measured at 100 fs for the $\mathbf{1 a}-\mathbf{C N}$ (blue) and $\mathbf{1 b}-\mathbf{C N}$ (orange) states. B. Decay kinetics, where the faster decay following excitation of $\mathbf{1 b}-\mathbf{C N}$ is evident. C. Comparison of FSRS averaged between 100 and 500 fs for 1a-CN and 1b-CN. D. Transient FSRS data for $\mathbf{1 b}-\mathbf{C N}$.

The weak $>700 \mathrm{~nm}$ absorbing Franck-Condon excited states decay in $<100$ fs to a dark state[2,3,6], which then relaxes to either 1a or back to 1b. Significantly, the decay of the dark state is clearly (Fig. 2B) dependent on the initial state excited, with that accessed from the metastable 1b-CN ground state decaying with a rate that exceeds that for the dark state originating in 1a-CN by a factor of 3; the same result is found for the unsubstituted form.[6] This result is not compatible with a common minimum between the two excited states, suggesting either a different intermediate is accessed depending on the initial structure, from which a different conical intersection is accessible. Alternatively the different excess energy distribution in the rapidly formed dark states may modify their decay pathways. The FSRS data (Fig. 2C) point to a difference in the structure for the two differently prepared dark states. Although many vibrational frequencies are common, the dominant higher frequency mode is shifted to higher wavenumber in $\mathbf{1 b}-\mathbf{C N}$ and there are a number of smaller changes in the $\mathrm{C}=\mathrm{C}$ stretch region (Fig 2C). Further, a low frequency mode $\left(380 \mathrm{~cm}^{-1}\right)$ is shifted or attenuated in $\mathbf{1 b}-\mathbf{C N}$. These results then suggest that distinct minima exist on the excited state surface for the two dark states, and that each decays with a different rate. Further characterisation will required the identification and assignment of the modes active in FSRS, perhaps through isotope exchange; such measurements are planned. In addition there is an urgent need for high level quantum chemical calculations of vibrational spectra associated with species on reactive excited state potential energy surfaces

1. B.L Feringa, J. Org. Chem., 6635, 72, 2007

2. J. Conyard, et al J. Amer. Chem. Soc. 9692, 136, 2014

3. C. R. Hall, et al J. Amer. Chem. Soc. 7408, 139, 2017

4. A. Kazaryan, et al J. Chem. Theory and Comp 2189, 7, 2011

5. X. Panget al, J. Phys.Chem. A 1240, 121, 2017

6. C. R. Hall, et al Angewante Chemie. Int. Ed. 6203, 57, 2018 